\title{
Compliance Modeling of a Compliant Stage with Symmetric Configuration
}

\author{
Yunsong $\mathrm{Du}^{1,2^{*}}$, Guohua Gao ${ }^{1,2}$ \\ ${ }^{1}$ Faculty of Materials and Manufacturing, Beijing University of Technology, Beijing, China \\ ${ }^{2}$ College of Mechanical Engineering and Applied Electronics Technology, Beijing University of Technology, Beijing, China \\ Email: *duyunsongwei@163.com
}

How to cite this paper: Du, Y.S. and Gao, G.H. (2020) Compliance Modeling of a Compliant Stage with Symmetric Configuration. Journal of Applied Mathematics and Physics, 8, 2500-2508.

https://doi.org/10.4236/jamp.2020.811185

Received: October 28, 2020

Accepted: November 23, 2020

Published: November 26, 2020

Copyright $\odot 2020$ by author(s) and Scientific Research Publishing Inc. This work is licensed under the Creative Commons Attribution International License (CC BY 4.0).

http://creativecommons.org/licenses/by/4.0/

\begin{abstract}
This paper presents the compliance modeling of a compliant stage with symmetric configuration. Empirical compliance equations for the circular flexure hinge are first introduced. Using the matrix method, the output compliance of a compliant stage with symmetric configuration is then obtained. Finally, the compliances derived from the proposed theoretical model and finite element analysis (FEA) are compared. It indicates that the results calculated by the theoretical model are in good agreement with those derived from FEA, which demonstrates the accuracy of the theoretical model.
\end{abstract}

\section{Keywords}

Flexure Hinge, Compliance Modeling, Compliant Stage, Symmetric Configuration

\section{Introduction}

Compliant mechanisms, composed of notch flexure hinges, have lots of advantages, such as no friction losses, no need for lubrication, ease of fabrication, compactness, and suitability for use in small-scale applications [1] [2]. Due to these properties, compliant mechanisms have been used in many fields, including medical engineering, aerospace engineering, and bioengineering [3]-[9]. Compliance is an important property for compliant mechanisms. It has large influence on the design, optimization, and control strategy. Due to these reasons, the compliance modeling of a typical compliant mechanism is investigated in this paper. For compliant mechanisms, most of them have symmetric configuration, and the purpose is to get excellent decoupled property. The compliant mechanism with decoupled property can be easier controlled, and thus possess high precision. 
Nowadays, lots of methods have been employed to obtain the compliance modeling of compliant mechanisms [10] [11] [12] [13] [14] including the pseudo rigid body method, the finite element analysis and the matrix method. Qu [15] analyzed the kinematic property of a micro-motion stage composed of flexure hinges. The stage is modeled by using the pseudo rigid body method. The circular flexure and wire flexure could be respectively modeled as a one-degreeof-freedom (1-DOF) revolute joint and a 3-DOF spherical joint, while the rest parts of the stage were regarded as rigid bodies. Finally, a prototype of the stage was fabricated, and the theoretical model was verified. Li [16] introduced a novel decoupled XY parallel micropositioning platform, coupled with uncomplicated structure and decoupled property. Its mechanism design and modeling was carried on by the matrix method. In order to verify the theoretical model, FEA was then employed, and the comparisons were discussed. Qin [17] proposed a novel compliant mechanism with 2-DOF. A computational optimization of the design based on FEA was first carried on. The compliance/stiffness of the proposed mechanism, the decoupled property of the leaf parallelograms, and the stress distribution at the lever mechanism were subsequently discussed and analyzed.

In this paper, the compliance modeling is analyzed in terms of the calculation errors of flexure hinges. The remaining sections of this paper are organized as follows. In Section 2, compliance equations for circular flexure hinge are discussed in terms of the influence of the stress concentration. In Section 3, a compliant stage with symmetric configuration is developed to analyze its output compliance, and the theoretical compliance model is derived from the matrix method. In Section 4, FEA is used to verify the accuracy of the proposed theoretical model. And the comparisons between FEA and theoretical model are carried on. Finally, conclusions are drawn in Section 5.

\section{Compliance of a Circular Flexure Hinge}

Compliant mechanisms are composed of several notch flexure hinges. These hinges are derived by drilling or milling two closely spaced holes, forming a certain cross-section cutout [18] [19]. For a circular flexure hinge, its cross-section is a circle, coupled with different main parameters. And its structure and the corresponding parameters can be illustrated in Figure 1, including the hinge thickness $t$, the hinge radius $R$, the rigid beam width $W$, the total height $H$, and the total depth $D$.

For a circular flexure hinge, the external load can be shown as Figure 2. Its origin of the coordinate system $x y z$ is located at the free end of the rigid beam. The $x$-axis is in the longitudinal direction of the hinge, while the $y$-axis is in the height direction. The flexure hinge has a load with six components: two shearing forces, $F_{y}, F_{z}$; two bending moments, $M_{y}, M_{z}$; a force along the $x$-axis, $F_{x}$; and a moment around the $x$-axis, $M_{x}$.

The deformation caused by the external load can be given as 


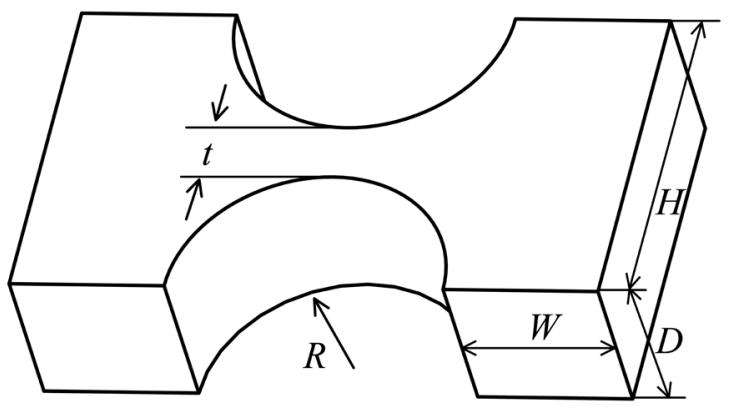

Figure 1. Geometrical parameters of a circular flexure hinge.

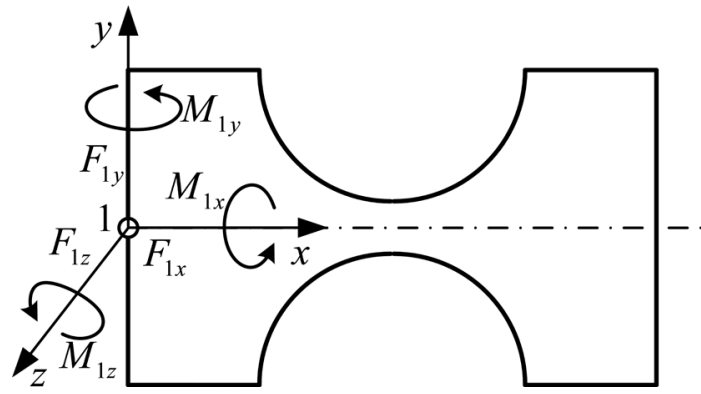

Figure 2. External load of a circular flexure hinge.

$$
\boldsymbol{u}=\boldsymbol{C F}
$$

For a flexure hinge, the spatial compliance matrix can be given as

$$
C_{s}=\left[\begin{array}{ccccc}
C_{x-F x} & 0 & 0 & 0 & 0 \\
0 & C_{y-F y} & 0 & 0 & C_{y-M z} \\
0 & 0 & C_{z-F z} & C_{z-M y} & 0 \\
0 & 0 & C_{\theta y-F z} & C_{\theta y-M y} & 0 \\
0 & C_{\theta z-F y} & 0 & 0 & C_{\alpha-M z}
\end{array}\right]
$$

where $C_{m-n}$ is the compliance in the direction of $m$ produced by the force/moment $n$.

The in-plane compliance matrix of a circular flexure hinge can be expressed as

$$
\boldsymbol{C}=\left[\begin{array}{ccc}
C_{x-F x} & 0 & 0 \\
0 & C_{y-F y} & C_{y-M z} \\
0 & C_{\alpha-F y} & C_{\alpha-M z}
\end{array}\right]
$$

The stress concentration has a large influence on the compliance calculation. Accordingly, the rigid beams residing next to the hinge should be considered when calculating compliance equations. In our earlier papers [20] [21], we have discussed the influence of the stress concentration on the compliance calculation of the common notch flexure hinges. Empirical compliance equations for the circular flexure hinge can be obtained based on the exponential model, and they can be expressed as

$$
C_{x-F x}=\frac{1}{E D(2 R+t)}\left[3.908 R\left(\frac{R}{t}\right)^{0.622}+2(R+W)\right]
$$




$$
\begin{gathered}
C_{\alpha-M z}=\frac{16}{E D t^{2}}\left(\frac{R}{t}\right)^{0.43} \\
C_{\alpha-F y}=\frac{16.58}{E D t}\left(\frac{R}{t}\right)^{1.427} \\
C_{y-F y}=\frac{17.3}{E D}\left(\frac{R}{t}\right)^{2.412}+\frac{\alpha E}{G} C_{x-F x} \\
C_{\alpha-F y}=\frac{16.55}{E D t}\left(\frac{R}{t}\right)^{1.429}
\end{gathered}
$$

where $\alpha$ is the shear coefficient, and $E$ is Young's modulus.

\section{Compliance Modeling}

Compliant mechanisms are composed of the parallel mechanism and serial mechanism. The parallel mechanism is advantageous over the serial mechanism. In addition, to get excellent decoupled property, the parallel mechanism is usually designed with symmetric configuration. Thus, the compliance of a compliant stage with symmetric configuration, coupled with decoupled property is analyzed in this paper. Note that the proposed compliant stage is composed of $4 \mathrm{pa}-$ rallel limbs, the left limb, the right limb, the upper limb and the lower limb. As shown in Figure 3, each limb contains two circular flexure hinges. Therefore,

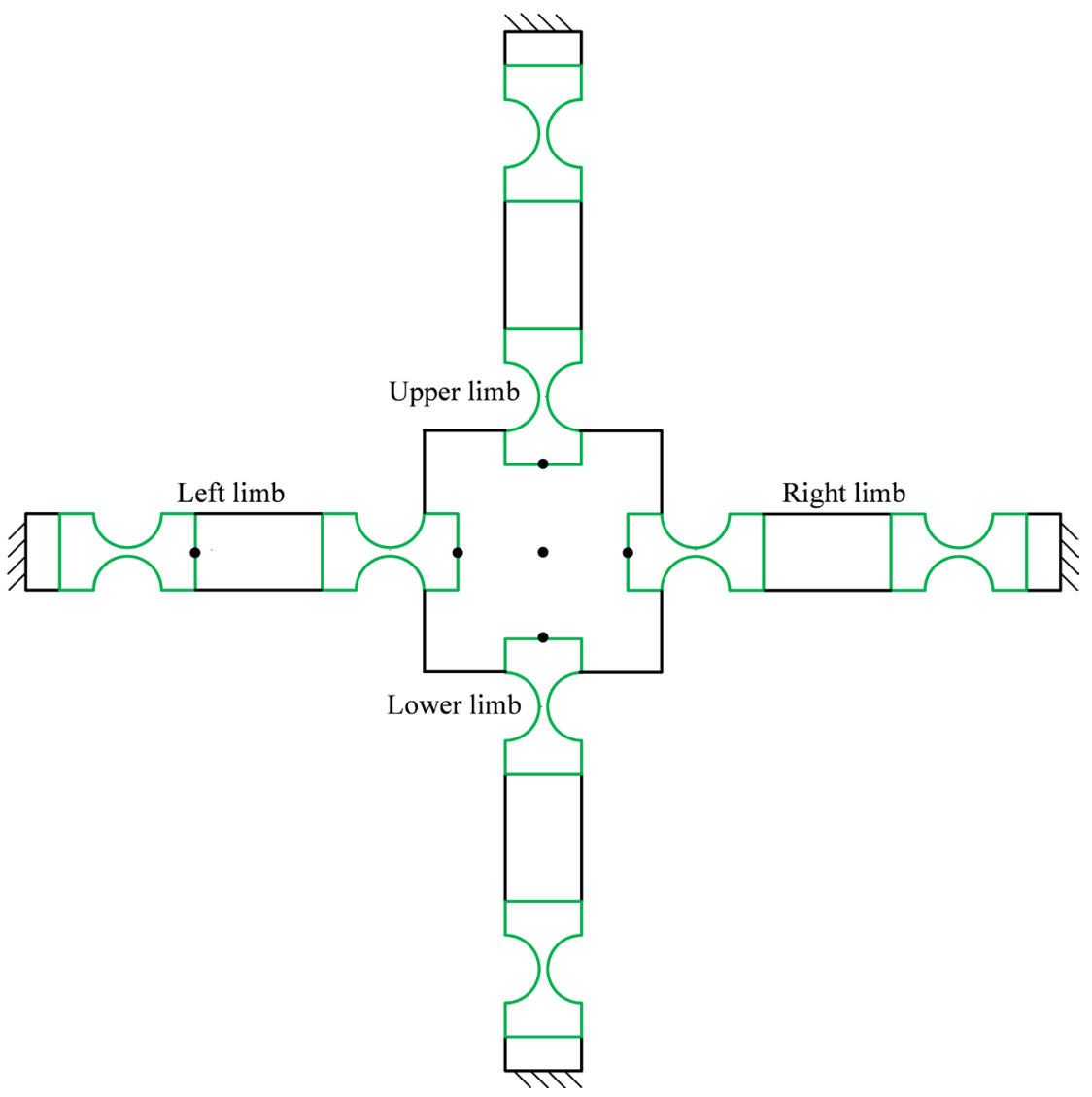

Figure 3. The compliant stage with symmetric configuration. 
the stage is composed of 8 circular flexure hinges, and their geometrical parameters are all the same.

As illustrated in Figure 4, the left limb of the compliant stage is first analyzed. Considering the influence of the stress concentration, the rigid beams of flexure hinges are considered. It is composed of the flexure hinge 1 and flexure hinge 2, and their local coordinate system $x_{1} y_{1} z_{1}$ and $x_{2} y_{2} z_{2}$ could be established at point $o_{1}$ and $o_{2}$, respectively. While the global coordinate system $x y z$ is established at point $o$, and parameters $L$ and $L_{1}$ are the key distances of the hinges.

Based on the matrix method, the compliance and the force/displacement transform matrix of the flexure hinge 1 at point $o$ located in the center of the output platform can be expressed as

$$
\begin{gathered}
\boldsymbol{C}_{1}=\boldsymbol{J}_{1} \boldsymbol{C} \boldsymbol{J}_{1}^{\mathrm{T}} \\
\boldsymbol{J}_{1}=\left[\begin{array}{ccc}
-1 & 0 & 0 \\
0 & 1 & -\left(L_{1}+L+w_{i}+2 R\right) \\
0 & 0 & -1
\end{array}\right]
\end{gathered}
$$

Similarly, the compliance and the force/displacement transform matrix of the flexure hinge 2 at point $o$ located in the center of the output platform can be expressed as

$$
\begin{gathered}
\boldsymbol{C}_{2}=\boldsymbol{J}_{2} \boldsymbol{C J}_{2}^{\mathrm{T}} \\
\boldsymbol{J}_{2}=\left[\begin{array}{ccc}
-1 & 0 & 0 \\
0 & 1 & -\left(L_{1}-w_{i}\right) \\
0 & 0 & -1
\end{array}\right]
\end{gathered}
$$

For the left limb, the compliances at point $o$ are obtained by summing all the contributions to the compliances in the corresponding directions of each individual flexure hinge, including flexure hinge 1 and hinge 2 , and thus the output compliance of the left limb at $o$ can be expressed as

$$
\boldsymbol{C}_{g l}=\boldsymbol{C}_{1}+\boldsymbol{C}_{2}=\boldsymbol{J}_{1} \boldsymbol{C J _ { 1 } ^ { \mathrm { T } }}+\boldsymbol{J}_{2} \boldsymbol{C J _ { 2 } ^ { \mathrm { T } }}
$$

The compliant stage is composed of 4 parallel limbs which are arranged $90^{\circ}$ apart (i.e., $\pi / 2 \mathrm{rad}$ ). According to the matrix method, the output compliance

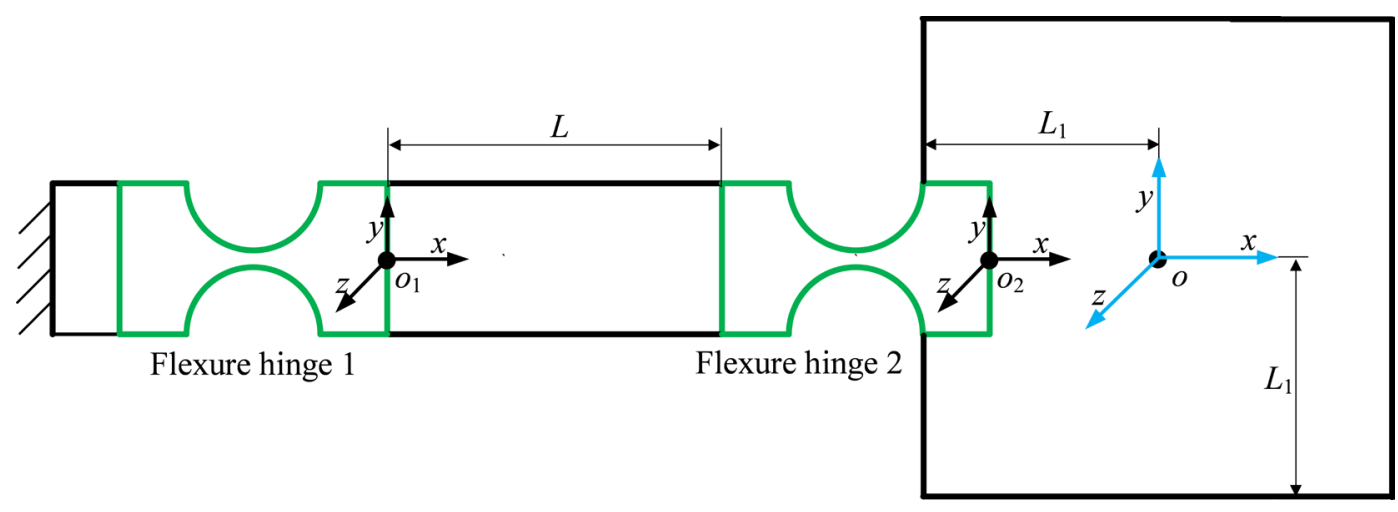

Figure 4. The left limb of the compliant stage. 
matrixes at point $o$ of the upper limb, right limb and lower limb, respectively, are

$$
\begin{aligned}
\boldsymbol{C}_{g s} & =\boldsymbol{J}_{d s} \boldsymbol{C}_{g l} \boldsymbol{J}_{d s}^{\mathrm{T}} \\
\text { where } \boldsymbol{J}_{d s} & =\left[\begin{array}{ccc}
\cos (\pi / 2) & -\sin (\pi / 2) & 0 \\
\sin (\pi / 2) & \cos (\pi / 2) & 0 \\
0 & 0 & 1
\end{array}\right] . \\
\text { where } \boldsymbol{J}_{d r} & =\left[\begin{array}{ccc}
\cos (\pi) & -\sin (\pi) & 0 \\
\sin (\pi) & \cos (\pi) & 0 \\
0 & 0 & 1
\end{array}\right] . \\
& \boldsymbol{J}_{d r} \boldsymbol{C}_{g l} \boldsymbol{J}_{d r}^{\mathrm{T}} \\
\text { where } \boldsymbol{J}_{d x} & =\left[\begin{array}{ccc}
\cos (3 \pi / 2) & -\sin (3 \pi / 2) & 0 \\
\sin (3 \pi / 2) & \cos (3 \pi / 2) & 0 \\
0 & 0 & 1
\end{array}\right] .
\end{aligned}
$$

Then, the total output compliance of the proposed compliance stage at point $o$ can be given as

$$
\boldsymbol{C}_{\text {total }}=\left\{\left(\boldsymbol{C}_{g l}\right)^{-1}+\left(\boldsymbol{C}_{g r}\right)^{-1}+\left(\boldsymbol{C}_{g s}\right)^{-1}+\left(\boldsymbol{C}_{g x}\right)^{-1}\right\}^{-1} .
$$

\section{Validation and Analysis}

The main compliances (the axial compliance $C_{x-F x}$ shear compliance $C_{y-F y}$ and bending compliance $C_{\alpha-M z}$ ) are first calculated by the proposed theoretical model and FEA, respectively. Considering the accuracy of FEA, it is used as a benchmark. All flexure hinges have equivalent geometrical parameters and material properties. In order to get a compact compliant stage coupled with the natural frequency more than $800 \mathrm{~Hz}$, a compliant stage is developed. Its hinge thickness is equal or more than $1 \mathrm{~mm}$ to process easily. Its geometrical parameters and material properties are given as $L=10 \mathrm{~mm}, L_{1}=15 \mathrm{~mm}, R=5 \mathrm{~mm}, t=1 \mathrm{~mm}$, $W=5 \mathrm{~mm}$, and $D=10 \mathrm{~mm}$. As shown in Figure 5 , a FEA model was generated using ANSYS 14.0. The rigid beams at the end of the limbs are fixed, including the surface $\mathrm{A}$, surface $\mathrm{B}$, surface $\mathrm{C}$ and surface $\mathrm{D}$. In addition, the forces $\left(F_{o x} F_{o n}\right.$ $M_{o z}$ ) are applied at point $o$. For similar mesh quality, the model with high mesh density has more elements and smaller simulation time steps, and thus increases calculation accuracy and computational costs, respectively. Thus, hexahedral meshes and appropriate mesh density are selected. There is a displacement output on point $O$, when imposing a force on the output platform.

The value calculated by theoretical model can be obtained based on Equation (17). In order to get the FEA value, the unite forces $\left(F_{o x}, F_{o y}, M_{o z}\right)$ can be applied, and the corresponding deformations can be obtained through the software. Of course, the calculation errors can be obtained based on Equation (18). The results 
derived from the theoretical model and FEA are compared in Table 1, and the maximum error is $4.7 \%$, while the minimum error is $3.5 \%$. It indicates that the proposed compliance theoretical model is accurate and reasonable.

$$
\text { Error }=\left(\left|C_{T H}-C_{F E A}\right| / C_{F E A}\right) \cdot 100 \% .
$$

where $C_{T H}$ is the theoretical value, and $C_{F E A}$ is the FEA value.

The vibrational modes and corresponding natural frequencies of the compliant stage are also analyzed by FEA. Figure 6 shows the first dominant mode shape for the compliant stage, and the first frequency is $975 \mathrm{~Hz}$.

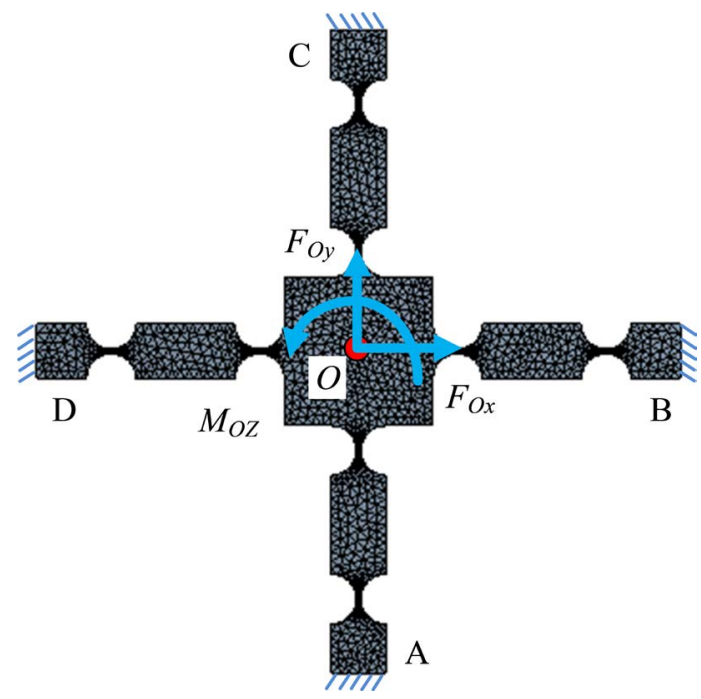

Figure 5. FEA model of the compliant stage.

Table 1. Errors between theoretical model and FEA.

\begin{tabular}{cccc}
\hline Errors (\%) & $C_{x-\mathrm{Fx}}$ & $C_{\mathrm{y}-\mathrm{Fy}}$ & $C_{\alpha-\mathrm{Mz}}$ \\
\hline 3.5 & 4.4 & 4.7 \\
\hline
\end{tabular}

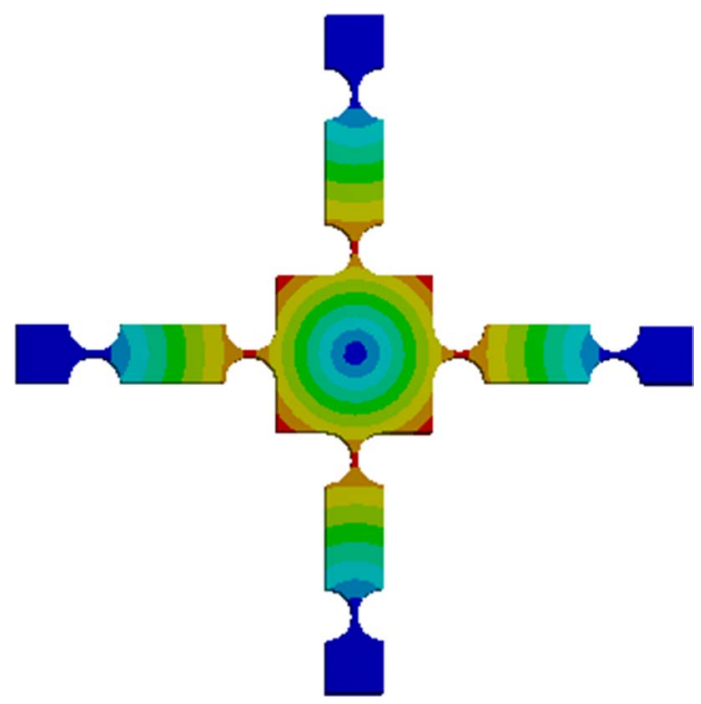

Figure 6. Mode shape of the compliant stage. 


\section{Conclusions}

In this paper, the output compliances of a compliant stage with symmetric configuration were derived in terms of the compliance calculation errors generated by stress concentration. These compliances were calculated by theoretical compliance equations and the matrix method, and subjected to FEA to validate the theoretical model. Based upon the results obtained, we can draw the following conclusions.

1) Compliance of a compliant mechanism can be obtained by accumulating deformations, forces and moments of flexure hinges.

2) Comparisons with the results obtained by FEA demonstrate that the proposed compliance theoretical model is valid.

\section{Acknowledgements}

This work was supported by Beijing Natural Science Foundation (3194044).

\section{Conflicts of Interest}

The authors declare no conflicts of interest regarding the publication of this paper.

\section{References}

[1] Polit, S. and Dong, J. (2011) Development of a High-Bandwidth XY Nanopositioning Stage for High-Rate Micro-/Nanomanufacturing. IEEE/ ASME Transactions on Mechatronics, 16, 724-733. https://doi.org/10.1109/TMECH.2010.2052107

[2] Yong, Y.K. and Lu, T.-F. (2009) Comparison of Circular Flexure Hinge Design Equations and the Derivation of Empirical Stiffness Formulations. Proceedings of the IEEE International Conference on Advanced Intelligent Mechatronics, Singapore, 14-17 July 2009, 510-515. https://doi.org/10.1109/AIM.2009.5229961

[3] Lai, L.-J., Gu, G.-Y. and Zhu, L.-M. (2012) Design and Control of a Decoupled Two Degree of Freedom Translational Parallel Micro-Positioning Stage. Review of Scientific Instruments, 83, 045105. https://doi.org/10.1063/1.3700182

[4] Li, Y., Xiao, S., Xi, L. and Wu, Z. (2014) Design, Modeling, Control and Experiment for a 2-DOF Compliant Micro-Motion Stage. International Journal of Precision Engineering and Manufacturing, 15, 735-744. https://doi.org/10.1007/s12541-014-0394-x

[5] Ouyang, P.R., Tjiptoprodjo, R.C., Zhang, W.J. and Yang, G.S. (2008) Micro-Motion Devices Technology: The State of Arts Review. The International Journal of Advanced Manufacturing Technology, 38, 463-478.

https://doi.org/10.1007/s00170-007-1109-6

[6] Zubir, M.N.M., Shirinzadeh, B. and Tian, Y. (2009) Development of a Novel Flexure-Based Microgripper for High Precision Micro-Object Manipulation. Sensors and Actuators A: Physical, 150, 257-266. https://doi.org/10.1016/j.sna.2009.01.016

[7] Li, Y. and Xu, Q. (2006) A Novel Design and Analysis of a 2-DOF Compliant Parallel Micromanipulator for Nanomanipulation. IEEE Transactions on Automation Science and Engineering, 3, 247-254. https://doi.org/10.1109/TASE.2006.875533

[8] Xu, Q. (2012) Design and Development of a Flexure-Based Dual-Stage Nanopositioning System with Minimum Interference Behavior. IEEE Transactions on Auto- 
mation Science and Engineering, 9, 554-563. https://doi.org/10.1109/TASE.2012.2198918

[9] Li, Y. and Xu, Q. (2008) Design of a New Decoupled XY Flexure Parallel Kinematic Manipulator with Actuator Isolation. IEEE/RSI International Conference on Intelligent Robots and Systems, Nice, 22-26 September 2008, 470-475.

[10] Hopkins, J.B. (2013) Designing Hybrid Flexure Systems and Elements Using Freedom and Constraint Topologies. Mechanical Sciences, 4, 319-331. https://doi.org/10.5194/ms-4-319-2013

[11] Dsouza, R.D., Navin, K.P., Theodoridis, T. and Sharma, P. (2018) Design, Fabrication and Testing of a 2 DOF Compliant Flexural Microgripper. Microsystem Technologies, 24, 3867-3883. https://doi.org/10.1007/s00542-018-3861-y

[12] Cai, K., Tian, Y., Wang, F., et al. (2017) Design and Control of a 6-Degree-of-Freedom Precision Positioning System. Robotics and Computer-Integrated Manufacturing, 44, 77-96. https://doi.org/10.1016/j.rcim.2016.08.005

[13] Clark, L., Shirinzadeh, B., Tian, Y., et al. (2016) Development of a Passive Compliant Mechanism for Measurement of Micro/Nanoscale Planar 3-DOF Motions. IEEE/ ASME Transactions on Mechatronics, 21, 1222-1232. https://doi.org/10.1109/TMECH.2015.2503728

[14] Shin, H.-P. and Moon, J.-H. (2018) Static and Dynamic Analyses of a 6-DOF U1tra-Precision Parallel Mechanism. International Journal of Precision Engineering and Manufacturing, 19, 1019-1026. https://doi.org/10.1007/s12541-018-0120-1

[15] Qu, J., Chen, W., Zhang, J. and Chen, W. (2016) A Piezo-Driven 2-DOF Compliant Micropositioning Stage with Remote Center of Motion. Sensors and Actuators A: Physical, 239, 114-126. https://doi.org/10.1016/j.sna.2016.01.025

[16] Li, Y. and Xu, Q. (2010) Development and Assessment of a Novel Decoupled XY Parallel Micropositioning Platform. IEEE/ ASME Transactions on Mechatronics, 15, 125-135. https://doi.org/10.1109/TMECH.2009.2019956

[17] Qin, Y., Tian, Y. and Zhang, D. (2012) Design and Dynamic Modeling of a 2-DOF Decoupled Flexure-Based Mechanism. Chinese Journal of Mechanical Engineering, 25, 688-696. https://doi.org/10.3901/CJME.2012.04.688

[18] Schotborgh, W.O., Kokkeler, F., Tragter, H. and van Houten, F.J.A.M. (2005) Dimensionless Design Graphs for Flexure Elements and a Comparison between Three Flexure Elements. Precision Engineering, 29, 41-47. https://doi.org/10.1016/j.precisioneng.2004.04.003

[19] Lobontiu, N. (2010) Compliant Mechanisms: Design of Flexure Hinges. CRC Press, Boca Raton.

[20] Li, T.M., Zhang, J.L. and Jiang, Y. (2015) Derivation of Empirical Compliance Equations for Circular Flexure Hinge Considering the Effect of Stress Concentration. International Journal of Precision Engineering and Manufacturing, 16, 1735-1743. https://doi.org/10.1007/s12541-015-0228-5

[21] Li, T., Du, Y., Jiang, Y. and Zhang, J. (2016) Empirical Compliance Equations for Constant Rectangular Cross Section Flexure Hinges and Their Applications. Mathematical Problems in Engineering, 2016, Article ID: 5602142. https://doi.org/10.1155/2016/5602142 